\title{
12-month outcomes of the US patient cohort in the SONATA pivotal IDE trial of transcervical ablation of uterine fibroids
}

This article was published in the following Dove Press journal:

International Journal of Women's Health

\author{
Joseph Hudgens' \\ D Alan Johns ${ }^{2}$ \\ Andrea S Lukes ${ }^{3}$ \\ David A Forstein ${ }^{4}$ \\ Dipak Delvadia ${ }^{5}$
}

'Department of Obstetrics and Gynecology, Eastern Virginia Medical School, Norfolk, VA, USA; ${ }^{2}$ Baylor Research Institute-Fort Worth, Fort Worth, TX, USA; ${ }^{3}$ Carolina Women's Research and Wellness Center, Durham, NC, USA; ${ }^{4}$ Department of Obstetrics and Gynecology, Touro College of Osteopathic Medicine, New York, NY, USA; ${ }^{5}$ Department of Obstetrics and Gynecology, Drexel University College of Medicine, Philadelphia, PA, USA
Correspondence: Joseph Hudgens

Eastern Virginia Medical School, 825

Fairfax Avenue, Suite 310, Hofheimer

Hall, Norfolk, VA 23507, USA

$\mathrm{Tel}+$ I 7574467979

Email hudgenjl@evms.edu
Objective: The prospective SONATA pivotal Investigational Device Exemption (IDE) trial was performed in the United States (US) and Mexico to examine the safety and effectiveness of transcervical fibroid ablation (TFA) in the treatment of symptomatic uterine fibroids. This is an analysis of 12-month clinical outcomes in the US cohort.

Methods: TFA with the Sonata ${ }^{\circledR}$ System was performed on women with symptomatic uterine fibroids. The 12-month co-primary endpoints were reduction in menstrual blood loss and freedom from surgical reintervention. Symptom severity, quality of life, patient satisfaction, safety, and reductions in uterine and fibroid volumes were also evaluated.

Results: One hundred twenty-five patients were enrolled and treated in the US. Both coprimary endpoints were achieved in this US-based cohort, as $65.3 \%$ of patients reported $\geq 50 \%$ reduction in menstrual bleeding and $99.2 \%$ of patients were free from surgical reintervention. Symptom improvement was noted by $97.4 \%$ of patients and $98.3 \%$ were satisfied. Ninety-five percent of patients reported reduced menstrual bleeding at 12 months, and $86.8 \%$ noted $>20 \%$ reduction. Significant mean improvements at 12 months were realized in both symptom severity and health-related quality of life (33.8 points and 45.8 points, respectively; all $P<0.0001)$. Mean maximal fibroid volume reduction per patient was $63.8 \%$. There was a $0 \%$ incidence of device related adverse events. Mean length of stay was $2.5 \mathrm{hrs}$ and $50 \%$ of patients returned to normal activity within 1 day.

Conclusion: This analysis of US patients in the SONATA pivotal IDE trial demonstrates results consistent with those in the full cohort. TFA with Sonata significantly reduced fibroid symptoms with a low surgical reintervention rate through 12 months. These results support the efficacy and safety of the Sonata system as a first-line treatment for women affected by symptomatic uterine fibroids.

Keywords: uterine leiomyoma, transcervical fibroid ablation; TFA, radiofrequency ablation, Sonata system

\section{Introduction}

The SONATA pivotal IDE trial ("SONATA trial;" ClinicalTrials.gov Identifier NCT02228174) was a prospective, longitudinal, multicenter, single-arm trial of transcervical fibroid ablation (TFA) with the Sonata ${ }^{\circledR}$ system in a cohort of women with symptomatic uterine fibroids. ${ }^{1}$ The primary endpoints at 12 months have been reported, and follow up is ongoing through 36 months. The overall trial population of 147 women were made up of patients from the US $(n=125)$ and Mexico $(n=22)$. Previous clinical results with the Sonata system demonstrated 
significant improvements in menstrual bleeding, quality of life and overall symptom severity in conjunction with excellent safety and surgical reintervention; total fibroid volume was reduced by $62 \%-67 \%$ at 12 months. ${ }^{1,2}$ This analysis examines the 12-month clinical outcomes of TFA with the Sonata system in the US patient cohort from the full SONATA trial.

\section{Materials and methods}

The Sonata system (Gynesonics, Inc; Redwood City, CA, USA), which has received United States Food and Drug Administration (FDA) clearance and Conformité Européene (CE) Mark, has been described in detail. ${ }^{2-5}$ Previously known as VizAblate ${ }^{\mathrm{TM}}$, the Sonata system (Figure 1) consists of a custom RF generator, a laptopbased ultrasound system with custom Graphical Guidance Software (GGS), and a single-use Radiofrequency Ablation (RFA) Handpiece integrally combined with a reusable Intrauterine Ultrasound (IUUS) Probe in a single treatment device. This system provides the gynecologist with a real-time, image-guided transcervical treatment option. Therapeutic radiofrequency energy is delivered to the fibroid according to a treatment cycle that is dependent on ablation size. Each ablation is scalable and determined graphically by the treating physician, maximizing the percentage of each fibroid to be ablated while avoiding thermal injury to adjacent viscera. The anesthesia choice for each patient may be individualized, as there is no requirement for general anesthesia; conscious sedation, and regional anesthesia (all with or without adjuvant paracervical blockade) were options provided within the SONATA clinical trial.

Premenopausal women between ages 25-50 with symptomatic uterine fibroids associated with heavy

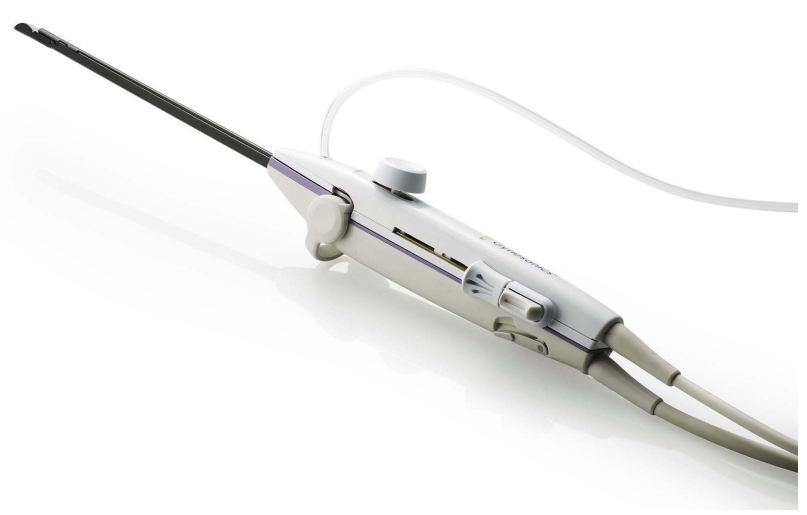

Figure I The Sonata treatment device, combining an intrauterine sonography probe with a radiofrequency ablation handpiece into a single integrated handpiece. menstrual bleeding were eligible for the SONATA trial if they had up to 10 myomata of International Federation of Gynecology and Obstetrics (FIGO) types 1, 2, 3, 4, and/or 2-5 (transmural) between 1-5 cm in diameter, experienced heavy menstrual bleeding as documented by a pictorial blood loss assessment chart (PBAC) score between 150500 , and had at least one fibroid that either indented (FIGO types 1, 2, 2-5) or abutted (FIGO type 3) the endometrial cavity. While type 5 and type 6 subserous myomata did not count towards the total number of fibroids, they could be ablated at the discretion of the investigator. Women were excluded if they desired fertility, had type 0 fibroids $1 \mathrm{~cm}$ or larger, had multiple endometrial polyps or any polyps $\geq 1.5 \mathrm{~cm}$ in diameter, a history of endometrial or fibroid ablation, prior uterine artery embolization (UAE), current tubal implants and clinically significant adenomyosis or substantial fibroid calcification.

Patients underwent screening that included transvaginal sonography, contrast-enhanced magnetic resonance (MR) imaging, assessment of menstrual bleeding via a pictorial blood loss assessment chart (PBAC), symptomatology and quality of life evaluation using the Uterine Fibroid Symptom and Quality-of-Life (UFS-QoL) Questionnaire, and a recording of baseline general health status using the EQ-5D (EuroQoL) questionnaire. All patients underwent cervical cancer and endometrial hyperplasia/carcinoma screening.

The US FDA approved the SONATA Investigational Device Exemption (IDE) trial in the US. Approvals were obtained from local and central institutional review boards (Table S1) prior to patient enrollment. The research was conducted according to the principles stated in the World Medical Association Declaration of Helsinki. Each patient provided her written informed consent to participate in the trial. All studies and records had protected health information deidentified.

The two co-primary endpoints of the SONATA trial, as required by the FDA, consisted of menstrual bleeding reduction at 12 months and freedom from surgical reintervention due to heavy menstrual bleeding (HMB) through 12 months. Overall study success required meeting the individual success criteria for both co-primary endpoints. Each patient was considered to have had a successful treatment outcome if she experienced $\geq 50 \%$ reduction in PBAC score with the score being $<250$. In terms of study success for this endpoint, the lower confidence limit (LCL) of the percentage of patient success was $\geq 45 \%$. For the 
surgical reintervention co-primary endpoint, patient success was defined as a lack of surgical reintervention for HMB due to treatment failure at 12 months, and its study success criterion was a LCL of the percentage of patient success $\geq 75 \%$.

Secondary endpoints included patient safety, reduction in total and perfused fibroid volumes at 12 months, change in the symptom severity score (SSS) and health-related quality of life (HRQOL) subscales of the UFS-QOL Questionnaire, overall patient treatment outcome using the Overall Treatment Effect (OTE) questionnaire, time to return to normal activity, patient satisfaction, change in general health outcome as determined by the EQ-5D questionnaire, pain and tolerance of the procedure, mean length of stay (LOS) and occurrence of pregnancy with pregnancy outcome. Treated patients were followed at 10 days, 30 days, 3 months, 6 months and at 12 months, with longer-term follow-up planned for 24 months and 36 months.

All statistical analyses were performed with SAS 9.3 (SAS, Cary, NC). Values were considered significant at the level of $\alpha=0.05$.

\section{Results}

Twenty-one clinical study sites in the US enrolled and treated 125 patients. Baseline patient and fibroid characteristics are provided in Table 1 and Table 2, respectively. Mean age of enrolled patients was $43.4 \pm 4.0$ years with a mean body mass index (BMI) of $29.5 \pm 6.4\left(\mathrm{~kg} / \mathrm{m}^{2}\right)$. Patients were $48 \%$ White, 39\% Black, and $17 \%$ Latina (Table 1).

Three patients were excluded from the Full Analysis Set population as they were diagnosed with menopause during the 12 months after TFA with the Sonata system, with a resultant inability to provide a PBAC diary at their 12month visits. Thus, the Full Analysis Set consists of 122 patients of the 125 patients. For the menstrual bleeding reduction endpoint, 121 of the 122 patients were included in this analysis, as one patient who underwent surgical reintervention prior to her 12-month visit was excluded from the analysis of this endpoint per the study statistical analysis plan. For the surgical reintervention endpoint evaluation, all 122 patients in the Full Analysis Set were included.

All 125 patients were treated in an outpatient setting, including 7 physician practice offices and 4 ambulatory care centers. Sixty-nine patients $(55.2 \%)$ were treated in an operating room, $33(26.4 \%)$ received their procedures in
Table I Baseline Patient Characteristics

\begin{tabular}{|l|l|}
\hline Characteristic & Results \\
\hline Age', N & 125 \\
Mean \pm SD & $43.5 \pm 4.0$ \\
Median & 44.0 \\
Min, Max & 33,50 \\
\hline Ethnicity, N & 125 \\
Hispanic or Latino & $21(16.8 \%)$ \\
Not Hispanic or Latino & $104(83.2 \%)$ \\
\hline Race ${ }^{2}, N$ & 125 \\
American Indian or Alaska Native & $3(2.4 \%)$ \\
Asian & $2(1.6 \%)$ \\
Black or African American & $49(39.2 \%)$ \\
Native Hawaiian or Other Pacific & $1(0.8 \%)$ \\
Islander & \\
White & $60(48.0 \%)$ \\
Other & $11(8.8 \%)$ \\
\hline BMI, N & 125 \\
Mean \pm SD & $29.5 \pm 6.4$ \\
Median & 28.0 \\
Min, Max & $18.0,49.8$ \\
\hline
\end{tabular}

Notes: 'Age in years. ${ }^{2}$ Subjects indicating multiple races are counted once under each race. Percentages may add to more than $100 \%{ }^{3}$ Calculated for those with both height and weight measurements $\left(\mathrm{kg} / \mathrm{m}^{2}\right)$

Table 2 Baseline PBAC and Fibroid Characteristics, Full Analysis Set $(N=122)$

\begin{tabular}{|l|l|}
\hline Characteristic & Results \\
\hline PBAC, N & 122 \\
Mean \pm SD & $293.5 \pm 96.1$ \\
Median & 281.0 \\
Min, Max & $150.2,498.3$ \\
\hline Total Fibroid Volume (cc), N & 117 \\
Mean \pm SD & $72.3 \pm 90.1$ \\
Median & 38.9 \\
Min, Max & $0.8,522.9$ \\
\hline Total Uterine Volume (cc), N & 122 \\
Mean \pm SD & $272.9 \pm 155.8$ \\
Median & 234.4 \\
Min, Max & $80.7,868.1$ \\
\hline
\end{tabular}

an ambulatory care center while 23 (18.4\%) patients were treated in a physician office. Seventy-four patients (59.2\%) received general anesthesia while 51 (40.8\%) had their procedures under conscious sedation. Paracervical blockade was co-administered as an ancillary local anesthetic modality in $54(43.2 \%)$ of patients. Mean length of stay 
Table 3 Change in PBAC Score by Visit

\begin{tabular}{|c|c|c|c|c|}
\hline PBAC Score & Baseline & 3 Months $^{a}$ & Change & $\%$ Change \\
\hline $\begin{array}{l}\text { N } \\
\text { Mean } \pm \text { SD } \\
\text { Median } \\
\text { Min, Max } \\
P\end{array}$ & $\begin{array}{l}|2| \\
293.9 \pm 96.3 \\
283.6 \\
150.2,498.3\end{array}$ & $\begin{array}{l}117 \\
175.9 \pm 110.3 \\
153.4 \\
11.7,647.8\end{array}$ & $\begin{array}{l}117 \\
-119.3 \pm 116.0 \\
-113.0 \\
-395.2,445.1 \\
<.0001\end{array}$ & $\begin{array}{l}117 \\
-38.9 \pm 39.1 \\
-44.4 \\
-96.5,219.6 \\
<.0001\end{array}$ \\
\hline $\begin{array}{l}\text { N } \\
\text { Mean } \pm \text { SD } \\
\text { Median } \\
\text { Min, Max } \\
P\end{array}$ & & $\begin{array}{l}6 \text { Months } \\
121 \\
146.3 \pm 102.7 \\
114.9 \\
11.7,519.9\end{array}$ & $\begin{array}{l}\text { Abs. Change } \\
|2| \\
-\mid 47.6 \pm 105.7 \\
-133.5 \\
-469.5,124.9 \\
<.000 \mid\end{array}$ & $\begin{array}{l}\% \text { Change } \\
|2| \\
-50.0 \pm 30.2 \\
-56.1 \\
-94.8,38.3 \\
<.000 \mid\end{array}$ \\
\hline $\begin{array}{l}\text { N } \\
\text { Mean } \pm \text { SD } \\
\text { Median } \\
\text { Min, Max } \\
P\end{array}$ & & $\begin{array}{l}\text { I2 Months } \\
12 \mid \\
14 \mid .3 \pm 107.2 \\
130.7 \\
0.0,902.2\end{array}$ & $\begin{array}{l}\text { Abs. Change } \\
|2| \\
-\mid 52.6 \pm 125.0 \\
-145.2 \\
-49 \mid .9,679.4 \\
<.000 \mid\end{array}$ & $\begin{array}{l}\text { \% Change } \\
|2| \\
-50.2 \pm 42.4 \\
-58 . \mid \\
-100.0,304.9 \\
<.000 \mid\end{array}$ \\
\hline
\end{tabular}

(including procedure time) was $2.5 \pm 1.3 \mathrm{hrs}$, with 91 of 125 patients $(72.8 \%)$ having a length of stay $\leq 3$ hrs. Median length of stay was $2.3 \mathrm{hrs}$.

Nearly all patients $(95 \% ; 115 / 121)$ experienced some reduction in menstrual bleeding at 12 months after TFA, with $86.3 \%$ experiencing reduced menstrual bleeding by 3 months. Regarding the 12-month PBAC timepoint, $65.3 \%(79 / 121)$ of patients (95\% CI 56.1-73.7\%) reported at least $50 \%$ reduction in menstrual bleeding, which met the endpoint success criterion that the LCL of the $95 \% \mathrm{CI}$ be $\geq 45 \%$. Detailed PBAC reduction results are provided in Table 3 .

The other co-primary endpoint, surgical reintervention for heavy menstrual bleeding at 12 months post-ablation, was also met. Only one patient underwent surgical intervention (elective hysterectomy for abnormal uterine bleeding) within the first 12 months of her post-treatment course. Thus, 99.2\% (95\% CI 94.3-99.9\%) of patients were free from surgical reintervention for heavy menstrual bleeding at 12 months after treatment with the Sonata system. This outcome met the endpoint success criterion that the LCL of the $95 \% \mathrm{CI}$ be $\geq 75 \%$. The percentage of patients achieving either co-primary endpoint did not significantly vary by ethnicity and were similar for White, Black and Latina patients.

Treatment with the Sonata system resulted in significant improvements in all patient-reported outcomes, beginning with the 3-month visit (the first post-treatment visit that included these questionnaires). As seen in Figure 2, patients reported a mean SSS reduction of 33.8 points $(\mathrm{N}=115)$ at 12 months post-procedure $(P<0.0001)$ and a mean increase of 45.8 points in HRQOL $(\mathrm{N}=115 ; P<0.0001)$. Regarding the OTE questionnaire, $97.4 \%$ of patients responding at 12 months (112/115) noted improvement in their fibroid symptoms, while $1.7 \%(2 / 115)$ reported no change in symptoms and $0.9 \%(1 / 115)$ noted a worsening of symptoms.

Patients reported significantly improved health status on the EQ-5D questionnaire at 12 months post-procedure. Self-reported scores on the EQ-5D questionnaire range from values of less than 0 , representing health states worse than "death", to a maximum score of 1.0 , representing "perfect health." At baseline, US patients in the SONATA trial had a mean overall health score of 0.73 $(\mathrm{N}=122)$. At 12 months $(\mathrm{N}=114)$, their mean EQ-5D score rose to $0.90(P<0.0001)$. Of note, health status significantly improved as early as 3 months after treatment with Sonata, with EQ-5D scores rose to 0.88 at 3 months (n=118; $P<0.0001)$.

Of 115 patients reporting at 12 months, $98.3 \%$ of patients expressed satisfaction with their treatment, and a similar percentage (96.5\%) would also recommend Sonata to a friend or family member. Specifically, $70.4 \%$ of reporting patients indicated that they were "very satisfied" with treatment, 18.3\% were "moderately 


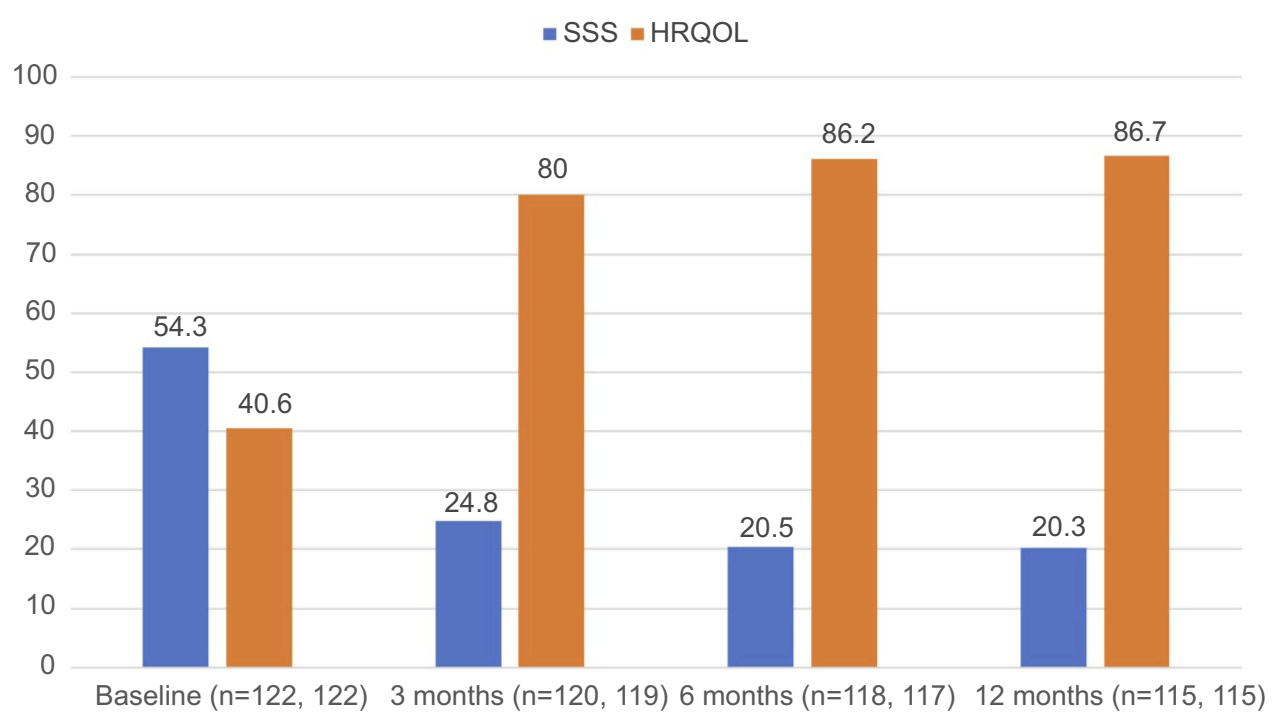

Figure 2 Improvements in uterine fibroid symptom and quality of life questionnaire subscales through 12 months in the FAS population (mean values). The SSS subscale demonstrates symptom reduction whereas the HRQoL subscale denotes increases in health-related quality of life. The results on both subscales signify improvement (all $P<0.0001)$.

satisfied," 9.6\% were "somewhat satisfied," $0.9 \%$ were "somewhat dissatisfied," and $0.9 \%$ were "moderately dissatisfied" at 12 months. No patients were "very dissatisfied" with their treatment. Similarly, 82.6\% (95/115) would "definitely recommend" treatment with Sonata, $13.9 \%$ would "probably recommend" it, and $3.5 \%$ would "probably not recommend" it at 12 months. No patient indicated that she would "definitely not recommend" the treatment.

Pain scores were evaluated on a scale from 0 ("No pain sensation") to 10 ("Most intense pain imaginable"). Mean procedural pain scores were $0.01 \pm 0.1$ for procedures under general anesthesia $(\mathrm{N}=74)$ and $0.6 \pm 1.6$ for procedures performed under conscious sedation $(\mathrm{N}=51)$. Recovery pain scores were $3.4 \pm 2.9$ for patients receiving general anesthesia and $2.5 \pm 2.7$ for those who were treated under conscious sedation. Overall, 98\% (122/125) of patients found treatment with the Sonata system to have been tolerable. Specifically, $63.2 \%(79 / 125)$ of patients reported the procedure to have been "Very Tolerable", 32\% (40/147) found the procedure to be "Moderately Tolerable", 2.4\% (3/147) characterized it as "Minimally Tolerable", and 2.4\% (3/147) said it was "Intolerable". Overall mean pain scores regardless of anesthesia choice were $0.2 \pm 1.0$ (range 0.0-7.0) during the procedure and $3.0 \pm 2.8$ (range 0.0-10.0) during recovery (reported from the time between procedure completion and discharge).
Table 4 Attributes of Ablated Fibroids*

\begin{tabular}{|c|c|}
\hline Procedure Parameter & \\
\hline Treated Fibroid Diameter, $\mathbf{N}$ & 392 \\
\hline$<1 \mathrm{~cm}$ & $20(5.1 \%)$ \\
\hline $1-2 \mathrm{~cm}$ & $144(36.7 \%)$ \\
\hline$>2-3 \mathrm{~cm}$ & $108(27.6 \%)$ \\
\hline$>3-4 \mathrm{~cm}$ & 69 (17.6\%) \\
\hline$>4 \mathrm{~cm}$ & $51(13.0 \%)$ \\
\hline Treated Fibroid Type, $\mathbf{N}$ & 392 \\
\hline Type I & $13(3.3 \%)$ \\
\hline Type 2 & $76(19.4 \%)$ \\
\hline Type 2-5 & 70 (I7.9\%) \\
\hline Type 3 & $102(26.0 \%)$ \\
\hline Type 4 & 89 (22.7\%) \\
\hline Type 5 & $39(9.9 \%)$ \\
\hline Type 6 & $3(0.8 \%)$ \\
\hline Number of Fibroids/Patient, $\mathbf{N}$ & 125 \\
\hline Mean \pm SD & $3.6 \pm 2.2$ \\
\hline Median & 3.0 \\
\hline Min, Max & $1.0,10.0$ \\
\hline Number of Treated Fibroids/Patient, $\mathbf{N}$ & 125 \\
\hline Mean \pm SD & $3.1 \pm 2.2$ \\
\hline Median & 3.0 \\
\hline Min, Max & $1.0,9.0$ \\
\hline Treated Fibroid Diameter $(\mathrm{cm}), \mathbf{N}$ & 392 \\
\hline Mean \pm SD & $2.5 \pm 1.2$ \\
\hline Median & 2.3 \\
\hline Min, Max & $0.3,6.5$ \\
\hline
\end{tabular}

Notes: *Determined via intrauterine sonography at the time of treatment $\mathrm{SD}$, standard deviation 
Table 4 summarizes characteristics of ablated fibroids. The mean maximal reduction in total leiomyoma volume per patient from baseline to 12 months was $63.8 \%$ $(\mathrm{N}=109)$. Of the 121 patients included in the analysis of the bleeding reduction co-primary endpoint, there were 33 patients $(27.3 \%)$ for whom a type 3 myoma was the sole qualifying fibroid type (ie, they did not have an indenting fibroid such as a type 2 myoma). Of these 33 patients, 22 $(66.7 \%)$ had at least a $50 \%$ reduction in PBAC score at 12 months. This is similar to the percentages of patients with indenting fibroids who achieved a $50 \%$ or greater reduction in PBAC score at 12 months (58.3\%, 67.4\% and $63.0 \%$ for patients with at least one type 1 fibroid, patients without any type 1 fibroids and at least one type 2 fibroid, and patients without any type 1 and type 2 fibroids and at least one type 2-5 fibroid, respectively).

Patients reported returning to normal daily activities in 2.1 \pm 2.3 days, and half of the patients returned to normal activity the day following the procedure. Patients with employment at the time of procedure indicated having returned to work 3.7 \pm 2.6 days post-procedure on average, with half of the patients returning to work within 3 days after the procedure. Patients resumed a normal diet at $0.7 \pm 1.3$ days, normal sleep at 0.8 \pm 1.7 days, and normal urinary and bowel functions at 0.2 \pm 0.7 days and $1.5 \pm 1.9$ days, respectively.

There were no occurrences $(0.0 \%)$ of device related adverse events, serious or otherwise. There was 1 procedure-related serious adverse events reported in a single subject $(0.8 \%)$. That single procedure-related serious adverse event involved a deep venous lower extremity thrombus diagnosed 15 days post-procedure, managed as an outpatient without sequelae.

As shown in Table 5, the results of the US cohort from the SONATA trial and that of the original cohort including patients from Mexico are provided.

\section{Discussion}

TFA with the Sonata system provides a safe and effective transcervical treatment for symptomatic uterine fibroids that uses RF ablation to treat all types of nonpedunculated fibroids. ${ }^{5}$ Because it contains an integral intrauterine sonography probe, Sonata can image, target and treat fibroids that are not accessible to other transcervical methods such as hysteroscopic morcellation or resectoscopy. Indeed, in the SONATA clinical trial, $77.3 \%$ of treated fibroids in the US cohort were not amenable to operative hysteroscopy, including intramural, transmural and subserous myomata. While it is true that some type 1 and type 2 submucous
Table 5 Outcomes of the Full and US-only Cohorts of the SONATA Pivotal IDE Trial

\begin{tabular}{|c|c|c|}
\hline Parameter & $\begin{array}{l}\text { SONATA } \\
\text { (US+Mexico) }\end{array}$ & $\begin{array}{l}\text { SONATA } \\
\text { (US) }\end{array}$ \\
\hline $\begin{array}{l}\geq 50 \% \text { reduction in menstrual } \\
\text { bleeding at } 12 \text { months }(\%)\end{array}$ & $64.8(\mathrm{~N}=142)$ & $65.3(\mathrm{~N}=121)$ \\
\hline $\begin{array}{l}\text { Freedom from surgical reinter- } \\
\text { vention at } 12 \text { months (\%) }\end{array}$ & $99.3(N=143)$ & $99.2(\mathrm{~N}=122)$ \\
\hline $\begin{array}{l}\text { Mean SSS reduction at } 12 \\
\text { months (points) }\end{array}$ & $32.1(\mathrm{~N}=135)$ & $33.8(\mathrm{~N}=115)$ \\
\hline $\begin{array}{l}\text { Mean HRQOL increase at } 12 \\
\text { months (points) }\end{array}$ & $43.7(\mathrm{~N}=134)$ & $45.8(\mathrm{~N}=115)$ \\
\hline $\begin{array}{l}\text { Percentage of patients reporting } \\
\text { improvement in overall fibroid } \\
\text { symptoms (OTE questionnaire) }\end{array}$ & $96.3(\mathrm{~N}=135)$ & $97.4(\mathrm{~N}=115)$ \\
\hline $\begin{array}{l}\text { Mean return to normal daily } \\
\text { activities (days) }\end{array}$ & $2.2(N=139)$ & $2.1(\mathrm{~N}=118)$ \\
\hline Mean return to work (days) & $3.6(N=111)$ & $3.7(\mathrm{~N}=96)$ \\
\hline Mean length of stay (hours) & $2.5(\mathrm{~N}=147)$ & $2.5(\mathrm{~N}=125)$ \\
\hline $\begin{array}{l}\text { Percentage of patients at least } \\
\text { "Somewhat Satisfied" at } 12 \\
\text { months }\end{array}$ & $97.1(\mathrm{~N}=135)$ & $98.3(\mathrm{~N}=\mid 15)$ \\
\hline $\begin{array}{l}\text { Percentage of patients who } \\
\text { would at least "Probably } \\
\text { Recommend" Sonata to a friend } \\
\text { or family member }\end{array}$ & $97.1(\mathrm{~N}=135)$ & $96.5(\mathrm{~N}=115)$ \\
\hline
\end{tabular}

myomata can be resected hysteroscopically, TFA obviates the inconvenience involved in removal of the resultant fibroid fragments, can address larger and deeper submucous fibroids that are less amenable to resection, and can be used in the same session to ablate other nonresectable fibroids without the need to also employ a resectoscope.

The US cohort represents $85 \%$ of patients treated in the SONATA trial, as 22 patients (15\%) in the 147-patient SONATA pivotal IDE trial were enrolled in Mexico. Clinical outcomes for US patients in the SONATA trial were consistent with those in the full (US+Mexico) SONATA trial population. The US cohort results also met both co-primary endpoints of the clinical trial.

Results from this US cohort of the SONATA trial as well as those from the full trial are consistent with the results of the previous FAST-EU trial, in which $64.6 \%$ of patients $(31 / 48)$ experienced a $>50 \%$ reduction in menstrual bleeding and reported a mean reduction of 35.3 points in their SSS, both at 12 months. ${ }^{1,3}$ In both the full and US-only cohorts of the SONATA trial, 95\% of patients had bleeding reduction, with $86.3 \%$ of patients experiencing bleeding reduction by 3 months. In the overall SONATA cohort of 147 patients, there were two 
reported procedure-related serious adverse events, one in Mexico and one in the US. There were no device related adverse events, serious or otherwise in the full SONATA or US cohorts. Patient satisfaction was similar in both groups.

The SONATA clinical trial was noteworthy for its inclusion of a wide variety of fibroid types (every FIGO fibroid type other than pedunculated fibroids [types 0 and 7] are treatable by the Sonata system) and increased number of treatable fibroids/patient compared with the prior FAST-EU clinical trial in Europe and Mexico. ${ }^{2,3}$ The SONATA trial included a robust patient selection process to minimize confounding factors, excluding patients with other etiologies of abnormal uterine bleeding such as anovulation and bleeding disorders. Furthermore, the study included a mix of patient-reported outcomes to complement the objective reintervention and bleeding primary endpoints.

Patients who expressly desired fertility were excluded. This was secondary to ethical reasons as SONATA was a pivotal safety and effectiveness investigational study. Nonetheless, the SONATA trial includes 3-year follow up of patients and the reporting of any pregnancy outcomes should they occur.

\section{Conclusion}

This analysis of US patients in the SONATA pivotal IDE trial demonstrates results consistent with those in the full cohort. TFA with Sonata significantly reduced fibroid symptoms with a low surgical reintervention rate through 12 months. These results support the efficacy and safety of the Sonata system as a first-line treatment for women affected by symptomatic uterine fibroids.

\section{Authors' Data Sharing Statement}

Will individual participant data be available (including data dictionaries)? No.

What data in particular will be shared: Not available.

What other study-related documents will be available? Not available.

When will data be available (start and end dates)? Not applicable.

By what access criteria will data be shared (including with whom, for what types of analyses, and by what mechanism)? Not applicable.

\section{Acknowledgments}

David B Toub, MD, MBA, Medical Director of Gynesonics, Inc, contributed to the preparation of this manuscript. Taraneh G Farazi, PhD, Vice-President of Clinical Affairs at Gynesonics contributed to the review of this manuscript. QST Consultations, Ltd provided biostatistical analysis for this study. The abstract of this paper was presented at the 2019 ACOG Annual Clinical and Scientific Meeting as a poster presentation. The poster's abstract was published in a supplement to the journal Obstetrics \& Gynecology (May 2019 - Volume 133 Issue - p 113S doi: 10.1097/01.AOG.0000558 838.56257.35 https://journals.lww.com/greenjournal/ Abstract/2019/05001/Transcervical_Radiofrequency_ Ablation_of_Uterine.391.aspx).

\section{Disclosure}

Joseph Hudgens reports he is one of the members at Speakers Bureau for Applied Medical. Andrea S Lukes reports grants from Gynesonics, Abbvie, Myovant, and Bayer, during the conduct of the study. David A Forstein reports grants from Gynesonics, Inc, during the conduct of the study. The institutions of each author received research support from Gynesonics, Inc. The authors report no other conflicts of interest in this work.

\section{References}

1. Chudnoff S, Guido R, Roy K, Levine D, Mihalov L, Garza-Leal JG. Ultrasound-guided transcervical ablation of uterine leiomyomas. Obstet Gynecol. 2019;133:13-22. doi:10.1097/AOG.0000000000 003032

2. Brölmann H, Bongers M, Garza-Leal JG, et al. The FAST-EU trial: 12-month clinical outcomes of women after intrauterine sonography-guided transcervical radiofrequency ablation of uterine fibroids. Gynecol Surg. 2016;13:27-35. doi:10.1007/s10397-0150915-3

3. Bongers M, Brölmann H, Gupta J, Garza-Leal JG, Toub D. Transcervical, intrauterine ultrasound-guided radiofrequency ablation of uterine fibroids with the VizAblate ${ }^{\circledR}$ system: three- and six-month endpoint results from the FAST-EU study. Gynecol Surg. 2015;12:6170. doi:10.1007/s10397-014-0873-1

4. Garza-Leal JG, Toub D, León IH, et al. Transcervical, intrauterine ultrasound-guided radiofrequency ablation of uterine fibroids with the VizAblate system: safety, tolerability, and ablation results in a closed abdomen setting. Gynecol Surg. 2011;8:327-334. doi:10.1007/s10397010-0655-3

5. Toub DB. A new paradigm for uterine fibroid treatment: transcervical, intrauterine sonography-guided radiofrequency ablation of uterine fibroids with the sonata system. Curr Obstet Gynecol Rep. 2017;6:67-73. doi:10.1007/s13669-017-0194-2 


\section{Supplementary material}

Table SI List of each Institutional Review Board (IRB)

\begin{tabular}{|l|l|}
\hline Institution Name & IRB \\
\hline Arizona Gynecology Consultants & Western Institutional Review Board, IRB \\
Advanced Women's Health Institute & Western Institutional Review Board, IRB \\
GW Medical Faculty Associates & Western Institutional Review Board, IRB \\
Christiana Care Health System & Christiana Care, IRB \\
KO Clinical Research & Western Institutional Review Board, IRB \\
Virtus Research Consultants & Western Institutional Review Board, IRB \\
University of Maryland School of Medicine & University of Maryland-Baltimore, IRB \\
Wayne State University Physician Group & Western Institutional Review Board, IRB \\
Mercy Clinic & Western Institutional Review Board, IRB \\
University of Mississippi Medical Center & University of Mississippi Medical Center, IRB \\
Women's Wellness Clinic & Western Institutional Review Board, IRB \\
Cooper University Hospital & Cooper Health System, IRB \\
Bosque Women's Care & Western Institutional Review Board, IRB \\
Albert Einstein School of Medicine-Montefiore & Biomedical Research Alliance of New York IRB (BRANY IRB) \\
Drexel University College of Medicine & Western Institutional Review Board, IRB \\
Magee-Women's Hospital & Western Institutional Review Board, IRB \\
PRISMA Health Upstate & Health Sciences South Carolina, IRB \\
Baylor Research Institute & Baylor Research Institute, IRB \\
Willowbend Health and Wellness & Western Institutional Review Board, IRB \\
Eastern Virginia Medical School & Eastern Virginia Medical School, IRB \\
Virginia Mason Medical Center & Western Institutional Review Board, IRB \\
\hline
\end{tabular}

\section{Publish your work in this journal}

The International Journal of Women's Health is an international, peerreviewed open-access journal publishing original research, reports, editorials, reviews and commentaries on all aspects of women's healthcare including gynecology, obstetrics, and breast cancer. The manuscript management system is completely online and includes a very quick and fair peer-review system, which is all easy to use. Visit http://www.dovepress.com/testimonials.php to read real quotes from published authors. 